Journal for ImmunoTherapy of Cancer

\title{
mutational burden, PD-L1 expression, and outcomes from immunotherapy in non-small cell lung cancer
}

Marcelo V Negrao (D) , ${ }^{1}$ Ferdinandos Skoulidis, ${ }^{1}$ Meagan Montesion, ${ }^{2}$ Katja Schulze, ${ }^{3}$ Ilze Bara, ${ }^{3}$ Vincent Shen, ${ }^{3} \mathrm{Hao} \mathrm{Xu},{ }^{3}$ Sylvia Hu, ${ }^{3}$ Dawen Sui, ${ }^{4}$ Yasir Y Elamin, ${ }^{1}$ Xiuning Le, ${ }^{1}$ Michael E Goldberg, ${ }^{2}$ Karthikeyan Murugesan, ${ }^{2}$ Chang-Jiun Wu, ${ }^{5}$ Jianhua Zhang, ${ }^{5}$ David S Barreto, ${ }^{6}$ Jacqulyne P Robichaux, ${ }^{1}$ Alexandre Reuben, ${ }^{1}$ Tina Cascone, ${ }^{1}$ Carl M Gay, ${ }^{1}$ Kyle G Mitchell (1) , ${ }^{7}$ Lingzhi Hong, ${ }^{1}$ Waree Rinsurongkawong, ${ }^{1}$ Jack A Roth, ${ }^{7}$ Stephen G Swisher, ${ }^{7}$ Jack Lee (D) , ${ }^{4}$ Anne Tsao, ${ }^{1}$ Vassiliki Papadimitrakopoulou, ${ }^{8}$ Don L Gibbons, ${ }^{1}$ Bonnie S Glisson, ${ }^{1}$ Gaurav Singal, ${ }^{2}$ Vincent A Miller, ${ }^{2}$ Brian Alexander, ${ }^{2}$ Garrett Frampton, ${ }^{2}$ Lee A Albacker, ${ }^{2}$ David Shames, ${ }^{3}$ Jianjun Zhang (D) , 1 John V Heymach ${ }^{1}$

To cite: Negrao MV, Skoulidis F, Montesion M, et al. Oncogenespecific differences in tumor mutational burden, PD-L1 expression, and outcomes from immunotherapy in non-small cell lung cancer. Journal for ImmunoTherapy of Cancer 2021;9:e002891. doi:10.1136/ jitc-2021-002891

- Additional supplemental material is published online only. To view, please visit the journal online (http://dx.doi.org/10. 1136/jitc-2021-002891).

Accepted 19 July 2021

Check for updates

(c) Author(s) (or their employer(s)) 2021. Re-use permitted under CC BY-NC. No commercial re-use. See rights and permissions. Published by BMJ.

For numbered affiliations see end of article.

\section{Correspondence to} Dr John V Heymach; JHeymach@MDAnderson.org

Dr Jianjun Zhang; jzhang20@mdanderson.org

\section{ABSTRACT}

Background Non-small cell lung cancer (NSCLC) patients bearing targetable oncogene alterations typically derive limited benefit from immune checkpoint blockade (ICB), which has been attributed to low tumor mutation burden (TMB) and/or PD-L1 levels. We investigated oncogenespecific differences in these markers and clinical outcome. Methods Three cohorts of NSCLC patients with oncogene alterations ( $n=4189$ total) were analyzed. Two clinical cohorts of advanced NSCLC patients treated with ICB monotherapy [MD Anderson (MDACC; $n=172$ ) and Flatiron Health-Foundation Medicine Clinico-Genomic Database (CGDB; $\mathrm{n}=894$ patients)] were analyzed for clinical outcome. The FMI biomarker cohort $(n=4017)$ was used to assess the association of oncogene alterations with TMB and PD-L1 expression.

Results High PD-L1 expression (PD-L1 $\geq 50 \%$ ) rate was $19 \%-20 \%$ in classic EGFR, EGFR exon 20 and HER2mutant tumors, and $34 \%-55 \%$ in tumors with $A L K, B R A F$ V600E, R0S1, RET, or MET alterations. Compared with KRAS-mutant tumors, BRAF non-V600E group had higher TMB (9.6 vs KRAS 7.8 mutations/Mb, $\mathrm{p}=0.003$ ), while all other oncogene groups had lower TMB $(p<0.001)$. In the two clinical cohorts treated with ICB, molecular groups with EGFR, HER2, ALK, ROS1, RET, or MET alterations had short progression-free survival (PFS; 1.8-3.7 months), while BRAFV600E group was associated with greater clinical benefit from ICB (CGDB cohort: PFS 9.8 months vs KRAS 3.7 months, HR 0.66, $\mathrm{p}=0.099$; MDACC cohort: response rate $62 \%$ vs $K R A S 24 \%$; PFS 7.4 vs KRAS 2.8 months, HR 0.36, $\mathrm{p}=0.026)$. KRAS G12C and non-G12C subgroups had similar clinical benefit from ICB in both cohorts. In a multivariable analysis, BRAFV600E mutation (HR 0.58, $p=0.041$ ), PD-L1 expression (HR 0.57, $p=0.022$ ), and high TMB (HR 0.66, $p<0.001)$ were associated with longer PFS.
Conclusions High TMB and PD-L1 expression are predictive for benefit from ICB treatment in oncogenedriven NSCLCs. NSCLC harboring BRAF mutations demonstrated superior benefit from ICB that may be attributed to higher TMB and higher PD-L1 expression in these tumors. Meanwhile EGFR and HER2 mutations and $A L K, R O S 1, R E T$, and MET fusions define NSCLC subsets with minimal benefit from ICB despite high PD-L1 expression in NSCLC harboring oncogene fusions. These findings indicate a TMB/PD-L1-independent impact on sensitivity to ICB for certain oncogene alterations.

\section{INTRODUCTION}

PD-1/PD-L1 immune checkpoint blockade (ICB) has revolutionized the treatment of many cancer types, including non-small cell lung cancer (NSCLC). ICB has the potential to induce durable responses; however, the response rate from single agent ICB is only $15 \%-20 \% .^{1-3}$ High PD-L1 expression $^{4-6}$ and high tumor mutational burden $(\mathrm{TMB})^{7-10}$ have been well documented to be associated with superior benefit from ICB in NSCLC. However, driver alterations in EGFR and $A L K$ oncogenes have been associated with cold immune microenvironment ${ }^{11}$ and poor outcomes from ICB therapy. ${ }^{1}{ }^{12-14}$ More recently, a study on the IMMUNOTARGET registry demonstrated distinct patterns of responses to ICB in NSCLC patients carrying rare canonical oncogene alterations. ${ }^{15}$ These data highlighted the critical impact of oncogene alterations on response to ICB. However, due to the scarcity of these rare 
oncogene-altered NSCLCs treated with ICB, the numbers of patients for certain oncogene groups were relatively small precluding definitive conclusions. Furthermore, the mechanisms underlying these distinct responses, and the impact of oncogene alterations on TMB, PD-L1 expression and on their predictive values are largely unknown, primarily due to lack of appropriate molecular and clinical data from rare oncogene-altered NSCLC patients treated with ICB.

To fill this void, we leveraged a large NSCLC cohort of 4189 patients, including 4017 NSCLC patients with oncogene alterations, TMB and PD-L1 data as well as two large registry cohorts consisting of 1066 NSCLC patients treated with ICB monotherapy to assess the impact of oncogene alterations on TMB, PD-L1 expression and clinical outcomes from ICB therapy.

\section{METHODS}

\section{Biomarker cohort}

\section{FMI biomarker cohort}

A total of 4017 NSCLC patients with molecular data including oncogene alterations, TMB and PD-L1 expression from the FMI FoundationCORE database were analyzed to assess the impact of oncogene mutations on TMB and PD-L1 (hereafter referred as FMI biomarker cohort).

\section{Clinical cohorts MDACC cohort}

GEMINI, a MD Anderson lung cancer database was queried. A total of 172 patients with advanced NSCLC whose tumor harbored oncogene alterations (see 'Oncogene alterations' section) and treated with single-agent ICB (pembrolizumab, nivolumab, atezolizumab, and durvalumab) between January 2014 and May 2018 were included. Data were collected through chart review, and dataset was locked on April 5, 2019. The GEMINI database is approved by the MD Anderson Cancer Center (MDACC) Institutional Review Board (IRB), and all patients signed informed consent.

\section{Clinico-Genomic database (CGDB) immunotherapy cohort}

The Flatiron-Foundation Medicine Inc CGDB, which is a clinically annotated subset of the FMI biomarker cohort, was queried and a total of 894 patients with advanced NSCLC harboring oncogene alterations (as defined in the 'Oncogene alterations' section) and treated with single-agent ICB between January 2011 and December 2018 were included (hereafter referred as CGDB immunotherapy cohort).

\section{CGDB chemotherapy cohort}

To understand whether the impact of oncogene alterations was specific to ICB, we also queried the FlatironFoundation Medicine Inc CGDB for patients with advanced NSCLC with oncogene alterations (as defined in the 'Oncogene alterations' section) and treated with combination or single-agent chemotherapy (hereafter referred as CGDB chemotherapy cohort). The study was IRB approved and included a waiver of informed consent. Data were collected from database repository, and dataset was locked on December 31, 2018.

This study was conducted in accordance with ethical guidelines including Declaration of Helsinki and US Common Rule.

\section{Oncogene alterations}

For the MDACC Cohort, genomic profiling information was obtained through Clinical Laboratory Improvement Amendments certified laboratory assay results annotated in the GEMINI database. For the CGDB and FMI biomarker cohorts, the information was obtained from respective data repositories and sequencing was performed by FoundationOne assay.

Oncogene alterations included in this study are: BRAF: codons 457-723, including V600E and non-V600E; EGFR exon 20: codons 762-823 (T790M mutation not included); HER2: codons 755 and 770-785; KRAS: codons 12, 13, 33, and 61; classic EGFR: exon 19 deletions and exon 21 p.L858R mutation ( \pm T790M mutation); MET: exon 14 skipping mutations; and ALK, ROS1, and RET: gene fusion/rearrangements.

\section{Tumor mutational burden}

For the CGDB cohorts and for the FMI biomarker cohort, TMB was determined through tissue-based nextgeneration sequencing (FoundationOne). TMB was defined as all mutations (including all non-synonymous and synonymous mutations with germline variants and driver mutations excluded) divided by the total covered exonic regions ${ }^{16}$ and presented as number of mutations/ megabase (Mb). High TMB is defined as $\geq 10$ mutations/ $\mathrm{Mb}$, corresponding to FMI diagnostic test definition of high TMB or $\geq 16$ mutations/Mb based on OAK and POPLAR trials. ${ }^{17}$ TMB data was not available for the MDACC cohort.

\section{PD-L1 staining}

For the MDACC cohort, PD-L1 staining was obtained from pathology reports. For the CGDB cohorts, PD-L1 status was obtained from data repository. For the FMI biomarker cohort, PD-L1 staining was assessed through immunohistochemistry using the 22C3 PharmDx assay (Dako North America). PD-L1 expression was quantified by tumor proportional score (TPS) and defined as positive $(\geq 1 \%)$ or negative $(<1 \%)$, and high $(\geq 50 \%)$ or low $(1 \%-49 \%) \cdot{ }^{5618}$

\section{Statistical analysis}

Progression-free survival (PFS) was defined as time from starting treatment until disease progression or death. Overall survival (OS) was defined as time from starting treatment until death. Patients without an event were censored at last follow-up. Left truncation method was applied to real-world PFS and OS analysis to adjust for 
situations where molecular profiling occurred after the start of treatment. ${ }^{19}$

Kaplan-Meier method was used to estimate PFS and OS, and differences were assessed by log-rank test. HRs and $95 \%$ CIs were determined through Cox proportional hazards model. Multivariable analysis was performed using the Cox regression method. Best response to ICB treatment determined through RECIST V.1.1 was available only for MDACC Cohort. The KRAS group was used as the reference comparator because: (1) it was the most commonly mutated oncogene in these cohorts of NSCLCs; and (2) KRAS-mutant NSCLCs constitute a heterogeneous patient population that have been shown to have similar prognosis compared with KRAS wildtype patients ${ }^{20-23}$ and to derive similar benefit from ICB therapy when compared with KRAS wild-type NSCLC patients. ${ }^{24}$

Differences in categorical variables were assessed through $\chi^{2}$ or Fisher's exact test with Bonferroni method for between group comparisons. Continuous variables were tested for normality using Shapiro-Wilk test, and Dunn's test with Benjamini-Hochberg correction was used for between group comparisons. Significance was established at $p$ value $\leq 0.05$. Statistical analysis was performed on GraphPad Prism V.7.0 (La Jolla, California, USA), IBM SPSS Statistics V.24.0 (Armonk, New York, USA), SAS (Cary, North Carolina, USA), and RStudio V.3.5.5 (Boston, Massachusetts, USA).

\section{RESULTS \\ TMB and PD-L1 are associated with clinical benefit from immunotherapy in oncogene-driven NSCLC}

We first analyzed the CGDB immunotherapy cohort (median follow-up 17.8 months (95\% CI 15.6 to 19.8)) to assess the predictive performance of TMB or PD-L1 for benefit from ICB in oncogene-driven NSCLC. We observed that higher TMB using either 10 or 16 mut/Mb as the cut-off was associated with longer PFS (TMB $\geq 10$ vs $<10 \mathrm{mut} / \mathrm{Mb}$ : HR $0.61,95 \%$ CI 0.50 to $0.75, \mathrm{p}<0.001$; TMB $\geq 16$ vs $<16$ mut/Mb: HR $0.52,95 \%$ CI 0.39 to 0.69 , $\mathrm{p}<0.001$ ), and longer $\mathrm{OS}$ ( $\mathrm{TMB} \geq 10 \mathrm{vs}<10 \mathrm{mut} / \mathrm{Mb}$ : HR $0.79,95 \%$ CI 0.64 to $0.97, \mathrm{p}<0.001$; TMB $\geq 16$ vs $<16$ mut/ Mb: HR $0.69,95 \%$ CI 0.51 to $0.92, \mathrm{p}<0.001$ ) consistent with previous findings. ${ }^{781017}$ Positive PD-L1 expression was associated with longer PFS (PD-L1 positive vs negative: HR $0.48,95 \%$ CI 0.30 to $0.77, \mathrm{p}<0.001$ ), but the difference did not reach statistical significance for OS (PD-L1 positive vs negative: HR $0.66,95 \%$ CI 0.39 to $1.10, \mathrm{p}=0.095$ ). In a multivariable analysis, higher TMB was significantly associated with longer PFS (TMB $\geq 10 \mathrm{vs}$ $<10 \mathrm{mut} / \mathrm{Mb}$ : HR $0.66,95 \%$ CI 0.53 to $0.82, \mathrm{p}<0.001$; TMB $\geq 16$ vs $<16$ mut/Mb: HR $0.56,95 \%$ CI 0.42 to 0.76 , $\mathrm{p}<0.001$ ) (online supplemental table $1 \mathrm{~A}, \mathrm{~B}$ ), and longer OS (TMB $\geq 10$ vs $<10 \mathrm{mut} / \mathrm{Mb}$ : HR 0.76 , 95\% CI 0.61 to $0.96, \mathrm{p}=0.019$; TMB $\geq 16$ vs $<16$ mut $/ \mathrm{Mb}$ : HR $0.66,95 \%$ CI 0.49 to $0.89, \mathrm{p}=0.006$ ) (online supplemental table $2 \mathrm{~A}, \mathrm{~B}$ ). PD-L1 expression was also associated with longer PFS
(TMB cut-off of 10: PD-L1 positive vs negative: HR 0.57 , $95 \%$ CI 0.35 to $0.92, p=0.022$ ) (online supplemental table $1 \mathrm{~A})$.

\section{Oncogene alterations were associated with distinct clinical outcomes from ICB therapy}

We next assessed the impact of oncogene alterations on clinical outcome from ICB treatment using 1066 oncogene-altered advanced NSCLC patients $(\mathrm{n}=172$ from MDACC cohort: median follow-up 29.1 months $(95 \%$ CI 26.2 to 33.6); and $n=894$ from CGDB immunotherapy cohort: median follow-up 17.8 months (95\% CI 15.6 to 19.8)) (online supplemental figure 1A,B). As outlined in online supplemental table 3, BRAF and $K R A S$ groups were enriched for smokers $(\mathrm{p}<0.001)$ in both MDACC cohort and CGDB immunotherapy cohort, while patients in the classic EGFR group received more prior treatment lines $(p<0.001)$ compared with other genomic groups, likely due to higher likelihood of prior exposure to TKIs $(\mathrm{p}<0.001)$. Since KRAS-mutant NSCLC constituted the largest population in both MDACC cohort $(\mathrm{n}=87)$ and CGDB immunotherapy cohort $(n=601)$ and have been previously shown to have similar prognosis compared with KRAS wild-type patients ${ }^{20-23}$ and to derive similar benefit from ICB therapy when compared with KRAS wild-type NSCLC patients, ${ }^{124}$ we used the KRAS group as the reference for outcome analysis.

In the MDACC cohort, the BRAF group had the longest PFS among all genomic groups (BRAF 7.4 vs KRAS 2.8 months, HR $0.36,95 \%$ CI 0.14 to $0.88, \mathrm{p}=0.026$ ) (figure 1A), while classic EGFR and HER2 groups had the shortest PFS (1.8 and 1.9 months, respectively) (figure 1A). The same trend remained in subsequent multivariable analysis adjusting for PD-L1 expression and smoking status (TMB data were not available for MDACC cohort) ( $\mathrm{p}=0.032$ for $B R A F$ group, $\mathrm{p}=0.022$ for Classic EGFR group, and $\mathrm{p}=0.116$ for HER-2 group, online supplemental table 4). Compared with the KRAS group, OS was numerically longer in $B R A F$ group, but the difference was not statistically significant (35.6 vs 16.8 months, HR 0.65 , $95 \%$ CI (0.26 to 1.63$), \mathrm{p}=0.363)$. Meanwhile patients with classic EGFR mutations had the shortest OS (11.3 months, 95\% CI 5.8 to 16.9, HR 2.01, $\mathrm{p}=0.006$ ) (figure 1B). Similar trends were observed in a multivariable analysis adjusting for PD-L1 expression, although the difference was not significant ( $B R A F$ vs $K R A S:$ HR 0.69, p=0.454; classic $E G F R$ vs KRAS: HR 1.49, $\mathrm{p}=0.263$; online supplemental table 5). Furthermore, the $B R A F$ group also had highest objective response rate (ORR) (62\%) among all oncogene groups (Fisher's exact test $\mathrm{p}<0.001$ ). Compared with the KRAS group, the common comparator, this difference was not statistically significant ( $62 \%$ vs $24 \%, p=0.364)$ (figure 1C). Importantly, two of three patients with $B R A F$ V600E mutations had recently progressed on vemurafenib and achieved durable responses at $35+$ and $20+$ months post treatment on ICB. There were seven patients harboring ALK fusion (n=2), RET fusion ( $\mathrm{n}=2$ ) or MET exon 14 skipping mutations $(n=3)$ in the MDACC cohort. Except for 


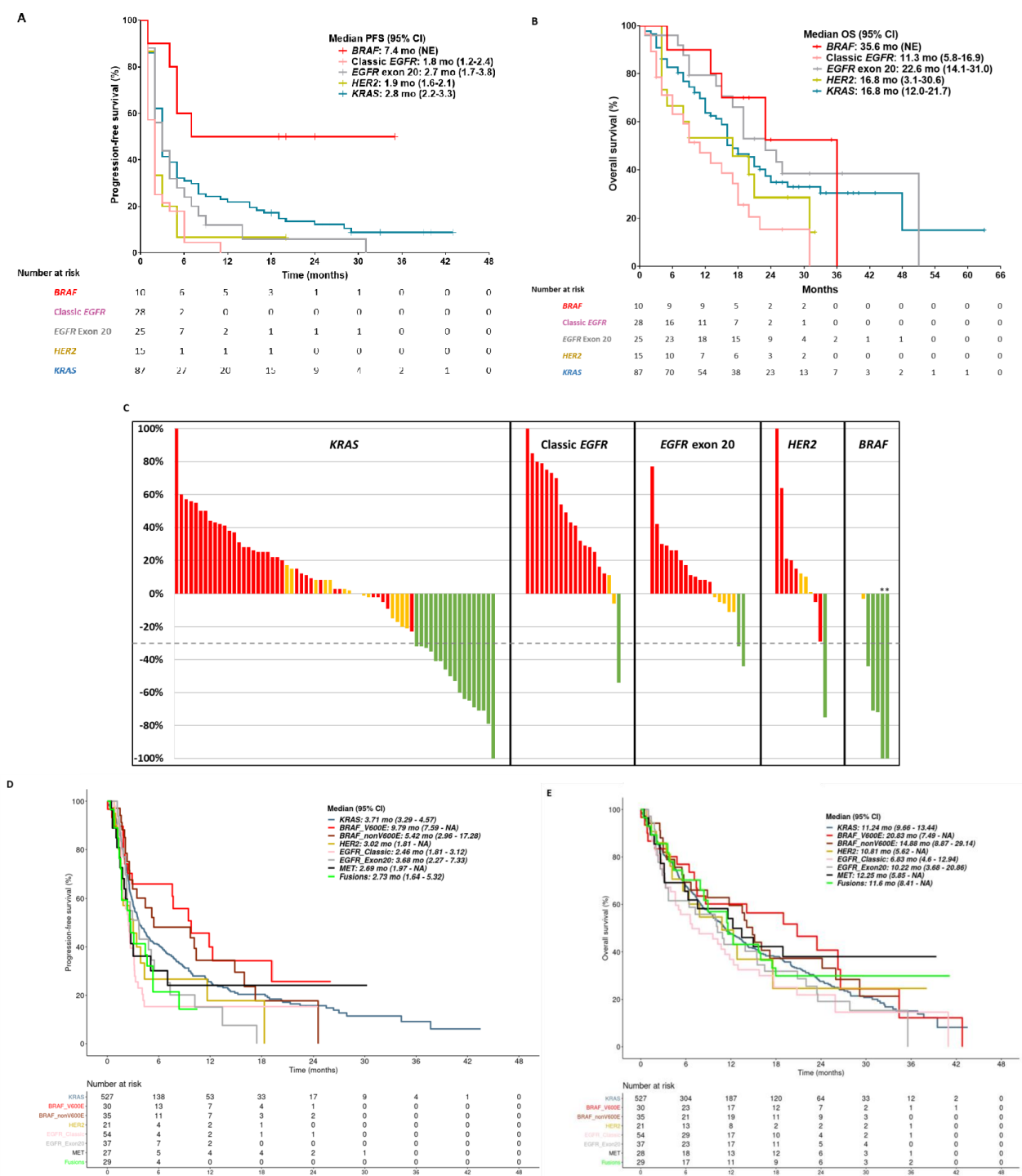

Figure 1 Clinical outcomes for oncogene-driven non-small cell lung cancers on treatment with single-agent PD-1/PD-L1 immune checkpoint inhibitor. (A) PFS in MDACC cohort; (B) OS in MDACC cohort; (C) waterfall plot for ORR in MDACC cohort for patients with measurable disease; (D) PFS in CGDB immunotherapy cohort; (E) OS in CGDB immunotherapy cohort.

*Patients harboring V600E alteration in the BRAF group. CGDB, Clinico-Genomic database; PFS, progression-free survival; OS, overall survival.

two patients with $M E T$ mutations who had stable disease, all remaining five patients had progressive disease as best response. Results for PFS, OS and ORR are summarized in table 1.

Consistent with the results from the MDACC Cohort, the BRAF groups in the CGDB immunotherapy cohort had the longest PFS (V600E 9.8 and non-V600E 5.4 months) (figure 1D and table 1) and OS (V600E 20.8 and non-V600E 14.9 months) (figure $1 \mathrm{E}$ and table 1) among all genomic groups although the difference did not reach statistical difference. Meanwhile, patients with classic EGFR mutations had significantly shorter PFS (figure 1D and table 1). Similar to classic EGFR group, patients with oncogene fusions (ALK: $\mathrm{n}=19$; ROS1: $\mathrm{n}=3$; RET: $\mathrm{n}=14$ ) had poor outcomes with a trend for shorter PFS compared with the KRAS group. The MET exon 14 mutation group $(\mathrm{n}=34)$ also had a short PFS of 2.7 months, but the difference was not significant compared with the KRAS group (HR 1.13, 95\% CI 0.69 to 1.84 , $\mathrm{p}=0.59$ ) (figure $1 \mathrm{D}$ and table 1 ). In a multivariable analysis adjusting for TMB, PD-L1 expression, and smoking status, BRAF V600E had longer PFS compared with the KRAS group (TMB cut-off of 10: HR $0.58,95 \%$ CI 0.35 to $0.98, \mathrm{p}=0.041$; TMB cut-off of 16 : HR $0.63,95 \%$ CI 0.38 to $1.05, \mathrm{p}=0.074$ ) (online supplemental table 1A,B).

KRAS-mutant group is the largest group in our cohorts, and we therefore assessed whether different KRAS-mutant alleles are associated with distinct clinical outcome from ICB treatment. As shown in online supplemental figure 2A-E, ORR and survival were similar between G12C, G12D and G12V subgroups.

To understand whether the impact of these oncogene alterations was only present on clinical outcome from ICB, we analyzed the CGDB chemotherapy cohort $(n=933)$ for 
clinical outcomes on treatment with chemotherapy across the same NSCLC oncogene groups (online supplemental figure $1 \mathrm{C}$, online supplemental table 6 ). These analyses demonstrated that the fusion group (ALK: $\mathrm{n}=39$; ROS1: $\mathrm{n}=12 ; R E T: \mathrm{n}=14$ ) had the longest OS with chemotherapy (27.2 months in fusion group vs 11.7 months in KRAS group, HR $0.50,95 \%$ CI 0.34 to $0.74, \mathrm{p}<0.001$ ) (online supplemental figure 3A). This difference remained significant in a multivariable analysis adjusting for prior TKI therapy (HR $0.48,95 \%$ CI 0.33 to $0.71, \mathrm{p}<0.001$ ). Otherwise, PFS or OS was not different between oncogene groups (online supplemental figure $3 \mathrm{~A}, \mathrm{~B}$ ).

\section{Oncogene-driven lung cancers show distinct patterns of PD- L1 expression and TMB}

We leveraged the 4017 NSCLC patients from FMI biomarker cohort to assess the impact of oncogene alterations on PD-L1 expression and TMB. High prevalence of PD-L1 positivity (TPS $\geq 1 \%$ ) was observed in MET (115/145, 79.3\%), RET (42/54, 77.8\%), BRAF V600E (89/118, 75.4\%), ROS1 (40/55, 72.7\%) and $A L K(136 / 194,70.1 \%)$ groups, and low prevalence was observed in tumors harboring EGFR exon $20(75 / 166$, 45.2\%), HER2 (50/105, 47.6\%), classic EGFR (exon 19 deletion and exon 21 L858R) $(368 / 732,50.3 \%)$, and $B R A F$ non-V600E (116/208, 55.8\%). Compared with KRASmutant NSCLCs, MET-mutant tumors had higher PD-L1 positivity rate (TPS $\geq 1 \%$ ), while EGFR-mutant tumors had significantly lower PD-L1 positivity rate (figure 2A) consistent with our previous findings. ${ }^{25}$ Furthermore, high PD-L1 expression (TPS $\geq 50 \%$ ) was common in tumors harboring alterations in MET $(80 / 145,55.2 \%), B R A F$ V600E (57/118, 48.3\%), ROS1 fusion (23/55, 41.8\%) and RET fusion $(20 / 54,37.0 \%)$. Among these, tumors harboring $M E T$ alterations had significantly higher prevalence of high PD-L1 expression (TPS $\geq 50 \%$ ), while classic EGFR, EGFR exon 20, and HER2 groups had lower prevalence of high PD-L1 compared with KRAS group (pairwise comparison vs KRAS $\mathrm{p}<0.050$ ) (figure $2 \mathrm{~B}$ ).

Compared with the KRAS group, TMB was higher for $B R A F$ non-V600E group (9.6 mut/Mb $(\mathrm{n}=208)$ vs 7.8 mut $/ \mathrm{Mb}(\mathrm{n}=2240)$; adjusted $\mathrm{p}=0.003)$, while all other oncogene groups had significantly lower TMB (adjusted $\mathrm{p}<0.001)$. Of note, $A L K(\mathrm{n}=194)$, classic EGFR $(\mathrm{n}=732)$, HER2 $(\mathrm{n}=105)$, RET $(\mathrm{n}=54)$, and ROS1 $(\mathrm{n}=55)$ groups had the lowest TMB with median $<3$ mut/ $\mathrm{Mb}$ (figure $2 \mathrm{C}$ and online supplemental table 7).

\section{DISCUSSION}

Oncogene drivers have been long known to determine the cancer biology and clinical behavior of NSCLC. In addition to the classic targetable genomic alterations such as activating EGFR mutations and $A L K$ fusions, more and more oncogene alterations defining distinct lung cancer groups are being discovered ${ }^{26}$ In the era of immuno-oncology, emerging evidence has demonstrated the profound impact of oncogene drivers on cancer 
A

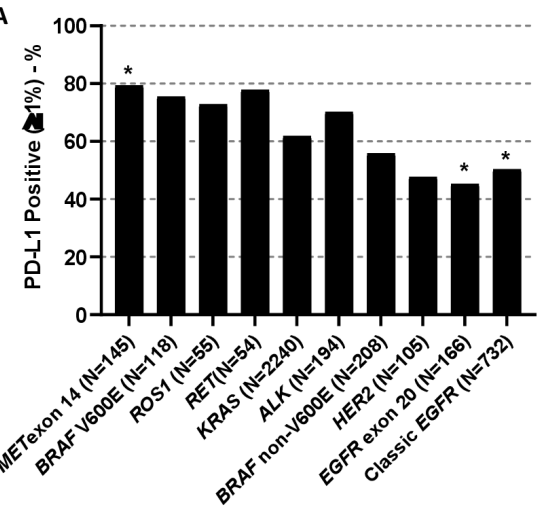

B

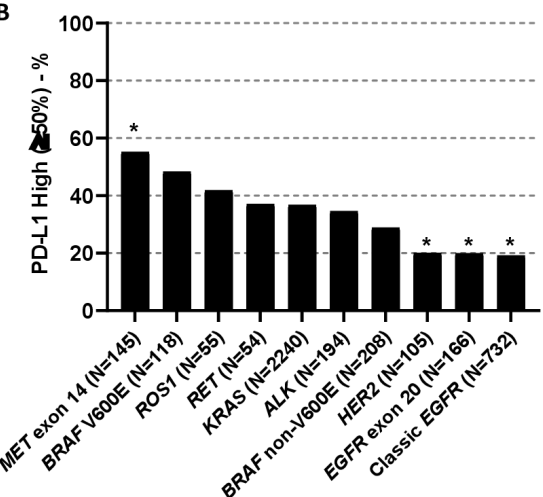

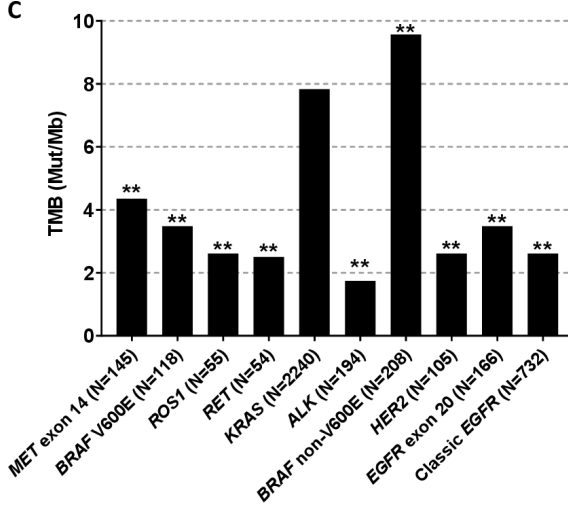

Figure 2 Oncogene-driven non-small cell lung cancers have distinct patterns of PD-L1 expression and TMB - FMI lung cancer database. (A) PD-L1 positivity rates; (B) high PD-L1 expression rates; (C) TMB. *Adjusted $\mathrm{p}<0.05$ versus KRAS group; ${ }^{*}$ adjusted $\mathrm{p}<0.01$ versus KRAS group. TMB, tumor mutational burden.

immune microenvironment ${ }^{1127}$ and benefit from ICB treatment. ${ }^{13152829}$ However, most of these intriguing findings are based on small cohorts because of the scarcity of data on certain genomic groups of NSCLC treated with single-agent ICB. ${ }^{13} 152829$ Furthermore, the association between oncogene drivers and established predictive markers for benefit from ICB such as PD-L1 and TMB has not been systemically studied. To fill this void, we leveraged the clinical and molecular data from over 4000 oncogene driven NSCLC patients and analyzed the association of oncogene drivers and TMB/PD-L1 expression as well as the benefit from ICB treatment.

Our study is consistent with previous reports demonstrating the impact of TMB and PD-L1 expression in clinical outcomes from ICB therapy in NSCLC but more specifically in a population of oncogene addicted NSCLC. Furthermore, BRAF-mutant NSCLC had high TMB and PD-L1 expression (figure 2A-C), which may make this group more sensitive to ICB therapy. These results suggest that ICB is a reasonable choice in the frontline setting for BRAF-mutant, PD-L1 + NSCLC given its potential to induce durable clinical benefit. It remains unclear why the outcomes reported for the $B R A F$ groups in the MDACC and CGDB immunotherapy cohorts are superior when compared with two previously published cohorts of BRAF-mutant NSCLC treated with ICB. ${ }^{15} 28$ Unfortunately, the unavailability of both TMB and PD-L1 data across the four cohorts precludes more definitive conclusions. Of note, $B R A F+M E K$ inhibition enhances immune responses in preclinical models ${ }^{30} 31$ and increases duration of response and PFS in combination with ICB in melanoma patients. ${ }^{32} 33$ Although it remains unknown if these combinations have activity in NSCLC, these findings suggest that ICB-TKI combinations could be explored in BRAF-mutant NSCLC.

KRAS G12C and non-G12C mutant NSCLC patients had similar clinical outcome in both analyzed immunotherapy cohorts highlighting that mutant allele was not associated with clinical outcome on treatment with ICB in KRAS-mutant NSCLC. These findings are consistent with prior work from our group and others highlighting that comutations (eg, STK11, KEAP1, and TP53), but not specific KRAS alleles, were associated with distinct subgroups with differing biological phenotypes and response to immunotherapy in KRAS-mutant NSCLC. ${ }^{15}$ 34-37 These results suggest that further therapeutic intervention is warranted to increase immunogenicity of KRAS-mutant NSCLC. The KRAS G12C inhibitor AMG510 resulted in a more inflamed tumor microenvironment and increased the efficacy of ICB in an immune competent mouse model.$^{38}$ Consequently, clinical investigation of KRAS G12C inhibitors and ICB combinations are ongoing.

Consistent with prior studies, our results also demonstrated that NSCLC patients harboring classic EGFR mutations had inferior outcomes from ICB. ${ }^{1} 91213$ 39-41 Additionally, we observed EGFR exon 20 and HER2 mutations were also associated with less benefit from singleagent ICB. This could be related to low TMB and low PD-L1 expression in these tumors. However, EGFR-mutant NSCLC tumors have been reported to have decreased proliferating and activated CD8 + Tcell infiltration, ${ }^{13}$ increased adenosine pathway signaling, as demonstrated by elevated expression of $\mathrm{CD} 73$ and adenosine $\mathrm{A} 1$ receptor, increased TGF- $\beta$ signaling, and lower IFN- $\gamma$ expression. ${ }^{27}$ Furthermore, our group has shown significantly lower $\mathrm{T}$ cell receptor (TCR) clonality implying less reactive TCR repertoire in EGFR-mutant NSCLC independent of TMB. ${ }^{1127}$ These findings indicate molecular mechanisms, other than low TMB, contribute to a cold immune microenvironment in EGFR-mutant NSCLC. Delineating these underlying mechanisms will allow development of effective immunotherapies for EGFR-mutant NSCLC. Contrary to EGFR and HER2, NSCLC harboring ALK/ROS1/RET fusions or $M E T$ exon 14 skipping mutations were found to have high PD-L1 expression although with low TMB. However, this did not translate to better clinical outcomes on ICB therapy as demonstrated by the short PFS and low $\mathrm{RR}$ in these groups. This suggests that oncogene-specific factors other than TMB and PD-L1 expression also impact clinical outcome from ICB therapy. 


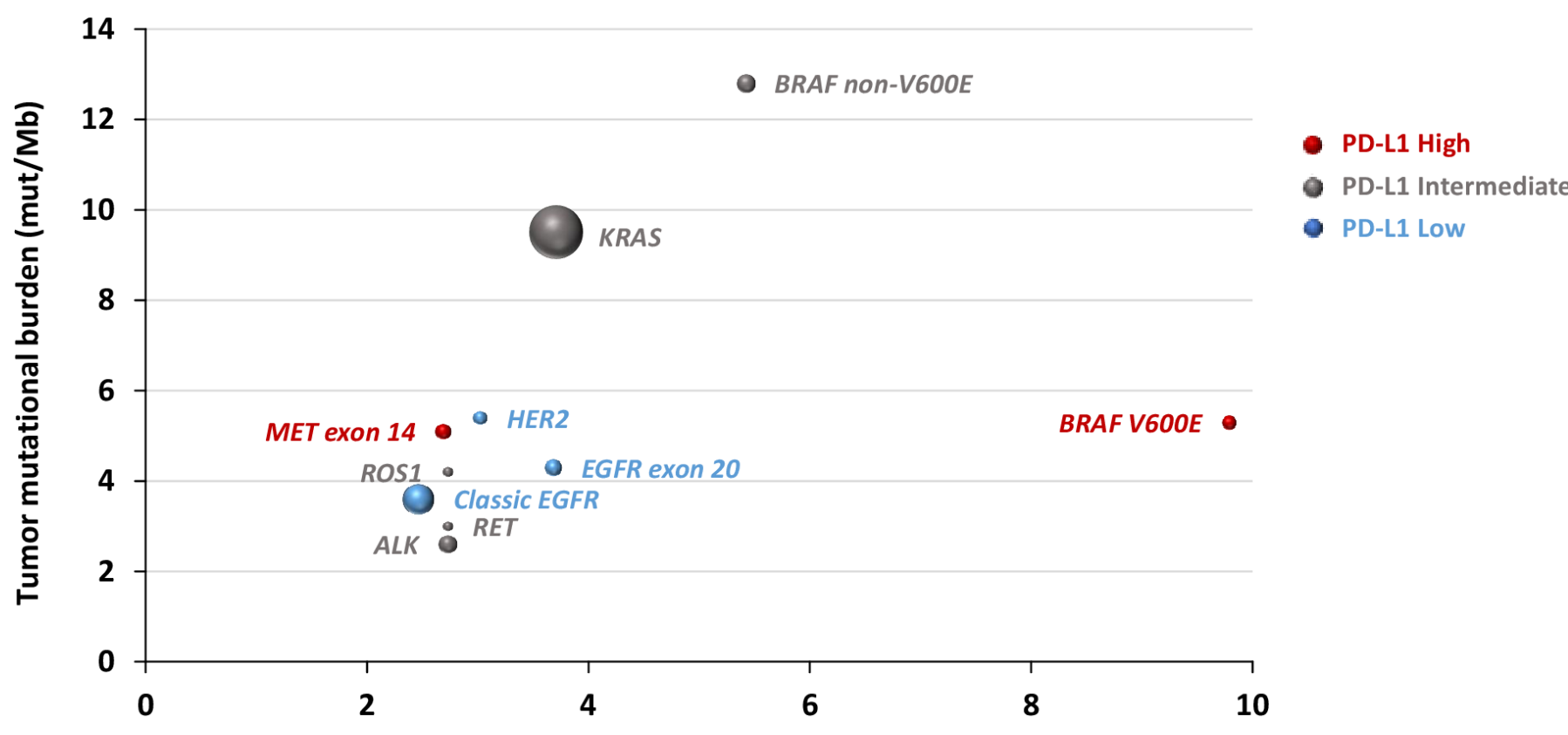

\section{Progression-free survival (months)}

Figure 3 Correlation between oncogene drivers and tumor mutational burden, progression-free survival and clinical outcome on immune checkpoint blockade therapy. Dot sizes are proportional to sample size; red: high PD-L1 expression; gray: intermediate PD-L1 expression; blue: low PD-L1 expression.

As a real-world study, our study has unavoidable limitations. First, the data are not consistent across different datasets, and many important data were missing. For example, tumor measurement was not available from the CGDB cohorts, which made estimation of ORR not feasible so that we were unable to validate the ORR from the MDACC cohort. In addition, the MDACC cohort did not have TMB data available, which limited further validation of the impact of TMB in predicting ICB outcome for oncogene-driven NSCLC. Second, we focused on NSCLC patients with oncogene drivers who received single-agent ICB. How these oncogene alterations impact the response and survival from ICB in combination with chemotherapy remains to be determined in future studies. Third, although we have over 4000 patients included, the sample size was still small, particularly for patients of certain genomic groups. For example, the number of patients in the BRAF-mutant group in the MDACC cohort was small, and we therefore had to combine V600E and non-V600E mutations for formal analysis, which limited us to validate the findings from the CGDB immunotherapy cohort and hampered further comparisons between V600E and nonV600E groups. Fourth, in the CGDB immunotherapy cohorts, PD-L1 status was missing in 76\%-92\% of patients and very few patients $(\mathrm{n}<5)$ had high TMB $(\geq 10$ or $\geq 16)$ across all oncogene groups with the exception of KRAS and BRAF non-V600E groups. This impaired our ability to analyze the predictive performance of PD-L1 or TMB in each oncogene group. Despite these limitations, our study creates a framework of groups of oncogene-driven lung cancers with distinct patterns of TMB and PD-L1 and different clinical outcomes on treatment with ICB that can be used for treatment decision in the clinic and for clinical trial design (figure 3).

\section{Author affiliations}

${ }^{1}$ Department of Thoracic/Head and Neck Medical Oncology, The University of Texas MD Anderson Cancer Center, Houston, Texas, USA

${ }^{2}$ Foundation Medicine Inc, Cambridge, Massachusetts, USA

${ }^{3}$ Genentech Inc, South San Francisco, California, USA

${ }^{4}$ Department of Biostatistics, The University of Texas MD Anderson Cancer Center, Houston, Texas, USA

${ }^{5}$ Department of Genomic Medicine, The University of Texas MD Anderson Cancer Center, Houston, Texas, USA

${ }^{6}$ Department of Radiology, Breast Imaging and Interventional Center, The George Washington University, Washington, DC, USA

${ }^{7}$ Department of Thoracic and Cardiovascular Surgery, The University of Texas MD Anderson Cancer Center, Houston, Texas, USA

${ }^{8}$ Pfizer Inc, New York, New York, USA

Correction notice This paper has been updated since first published to amend corresponding author details.

Contributors Conception and design: MVN, KS, IB, SH, XL, JPR, AR, JL, LAA, DS, JZ, JVH. Development of methodology: MVN, FS, MM, KS, IB, VS, HX, SH, DS, MEG, KM, C-JW, JZ, DSB, JPR, AR, TC, CMG, KGM, LH, WR, JL, LAA, DS, JZ, JVH. Acquisition of Data: MVN, FS, MM, KS, IB, VS, HX, SH, DS, YYE, XL, MEG, KM, C-JW, JZ, DSB, TC, CMG, KGM, LH, WR, GS, VAM, BMA, GF. Analysis and interpretation of data all authors. Writing, review and or revision of the manuscript: all authors. Administrative, technical, or material support: MVN, FS, MM, KS, IB, VS, HX, SH, DS, YYE, XL, MEG, KM, C-JW, JZ, DSB, JPR, AR, TC, CMG, KGM, LH, WR, GS, JL, AST, VP, DLG, BSG, GS, VAM, BMA, GMF, LAA, DSS, JZ, JVH.

Funding The authors would like to acknowledge funding support from University of Texas MD Anderson Lung Moon Shot Program and the MD Anderson Cancer Center Support Grant P30 CA01667, NIH R01 CA190628, NIH/NCI R01 CA20515001, Cancer Prevention \& Research Institute of Texas RP160652, UT Lung SPORE P50 CA70907, Rexanna's Foundation for Fighting Lung Cancer, Bruton Endowed Chair in Tumor Biology, Standing Fund for EGFR inhibitor resistance, the Hallman fund, Fox Lung EGFR Inhibitor Fund, and The Gil and Dody Weaver Foundation (to $\mathrm{JVH})$. 
Competing interests MVN is a consultant for Mirati and Merck/MSD, and reports funding to the institution from Mirati, Novartis, Pfizer, Ziopharm, AstraZeneca, and Checkmate; MM is an employee of Foundation Medicine, and owns stock in Roche; KS is an employee of Genentech, and owns stock in Roche; IB is an employee of Genentech, owns stock in Roche; VS is an employee of Roche, and owns stock in Roche; HX is an employee of Roche, and owns stock in Roche; HS is an employee of Genentech, and owns stock in Roche; XL is a Consultant for Eli Lilly, AstraZeneca, and EMD Serono; MEG is a former employee of Foundation Medicine; KM is an employee of Foundation Medicine, and owns stock in Roche; JPR reports licensing/ royalties from Spectrum Pharmaceuticals; TC has received speaker's fees from the Society for Immunotherapy of Cancer and Bristol-Myers Squibb, receives consulting fees from Medlmmune and Bristol-Myers Squibb, and reports research funding to MD Anderson Cancer Center from Boehringer Ingelheim, Medlmmune and Bristol-Myers Squibb; AT reports research grants from Eli Lilly, Millennium, Polaris, Genentech, Merck, Boehringer-Ingelheim, BMS, Ariad, Epizyme, Seattle Genetics, Takeda, and EMD Serono; advisory board member for BMS, Eli Lilly, Genentech, Roche, Novartis, Ariad, EMD Serono, Merck, Seattle Genetics, Astra-Zeneca, Boehringer-Ingelheim, Sellas Life Science, Takeda, Epizyme, and Huron; VP is an employee of Pfizer; DLG reports research grants from AstraZeneca, Jenssen R \& D, Takeda, Ribun Therapeutics; and honoraria member of Senofi, AstraZeneca, Ribun Therapeutics; GS is an employee of Foundation Medicine, and owns stock in Roche; VAM is an employee of Foundation Medicine, and owns stock in Roche; BA is an employee of Foundation Medicine, and owns stock in Roche; GF is an employee of Foundation Medicine, and owns stock in Roche; LAA is an employee of Foundation Medicine, and owns stock in Roche; DS is an employee of Genentech, and owns stock in Roche; JZ reports research funding from Merck, Johnson and Johnson, and consultant fees from BMS, Johnson and Johnson, AstraZeneca, Geneplus, OrigMed, Innovent outside the submitted work; JVH reports research grants from $\mathrm{NIH} / \mathrm{NCl}$, American Cancer Society, Cancer Prevention \& Research Institute of Texas, AACR Johnson \& Johnson Lung Cancer, AZ, Spectrum, Checkmate Pharmaceuticals; Advisory Committees - AZ, BMS, GSK, Guardant Health, Kairos Venture Investments, BrightPath Biotherapeutics Hengrui Therapeutics, Eli Lilly, Spectrum, EMD Serono, Roche, Foundation One Medicine; Royalties \& Licensing - Spectrum \& Bio-Tree Systems, Inc.

\section{Patient consent for publication Not required.}

Ethics approval This study was IRB approved and conducted in accordance with ethical guidelines including Declaration of Helsinki and US Common Rule.

Provenance and peer review Not commissioned; externally peer reviewed.

Data availability statement All data relevant to the study are included in the article or uploaded as supplementary information.

Supplemental material This content has been supplied by the author(s). It has not been vetted by BMJ Publishing Group Limited (BMJ) and may not have been peer-reviewed. Any opinions or recommendations discussed are solely those of the author(s) and are not endorsed by BMJ. BMJ disclaims all liability and responsibility arising from any reliance placed on the content. Where the content includes any translated material, BMJ does not warrant the accuracy and reliability of the translations (including but not limited to local regulations, clinical guidelines, terminology, drug names and drug dosages), and is not responsible for any error and/or omissions arising from translation and adaptation or otherwise.

Open access This is an open access article distributed in accordance with the Creative Commons Attribution Non Commercial (CC BY-NC 4.0) license, which permits others to distribute, remix, adapt, build upon this work non-commercially, and license their derivative works on different terms, provided the original work is properly cited, appropriate credit is given, any changes made indicated, and the use is non-commercial. See http://creativecommons.org/licenses/by-nc/4.0/.

\section{ORCID iDs}

Marcelo V Negrao http://orcid.org/0000-0001-8938-6699

Kyle G Mitchell http://orcid.org/0000-0002-4386-3258

Jack Lee http://orcid.org/0000-0001-5469-9214

Jianjun Zhang http://orcid.org/0000-0001-7872-3477

\section{REFERENCES}

1 Borghaei H, Paz-Ares L, Horn L, et al. Nivolumab versus docetaxel in advanced Nonsquamous Non-Small-Cell lung cancer. N Engl J Med 2015;373:1639:1627-39. doi:10.1056/NEJMoa1507643

2 Gettinger S, Horn L, Jackman D, et al. Five-Year follow-up of nivolumab in previously treated advanced non-small-cell lung cancer: results from the CA209-003 study. J Clin Oncol 2018;36:1675-84.
3 Garon EB, Hellmann MD, Rizvi NA, et al. Five-Year overall survival for patients with advanced Non-Small-Cell lung cancer treated with pembrolizumab: results from the phase I KEYNOTE-001 study. J Clin Oncol 2019:37:2518-27. doi:10.1200/JCO.19.00934

4 Reck M, Rodríguez-Abreu D, Robinson AG, et al. Pembrolizumab versus chemotherapy for PD-L1-positive non-small-cell lung cancer. N Engl J Med 2016;375:1823-33. doi:10.1056/NEJMoa1606774

5 Garon EB, Rizvi NA, Hui R. Pembrolizumab for the treatment of NonSmall-Cell lung cancer. N Engl J Med Overseas Ed 2015;372:2028. doi:10.1056/NEJMoa1501824

6 Gandhi L, Rodríguez-Abreu D, Gadgeel S, et al. Pembrolizumab plus chemotherapy in metastatic Non-Small-Cell lung cancer. $N$ Engl $J$ Med 2018;378:2078-92. doi:10.1056/NEJMoa1801005

7 Hellmann MD, Ciuleanu T-E, Pluzanski A, et al. Nivolumab plus ipilimumab in lung cancer with a high tumor mutational burden. $N$ Engl J Med 2018;378:2093-104. doi:10.1056/NEJMoa1801946

8 Rizvi NA, Hellmann MD, Snyder A, et al. Mutational landscape determines sensitivity to PD-1 blockade in non-small cell lung cancer. Science 2015;348:124-8. doi:10.1126/science.aaa1348

9 Rizvi H, Sanchez-Vega F, La K, et al. Molecular determinants of response to anti-programmed cell death (PD)-1 and antiprogrammed death-ligand 1 (PD-L1) blockade in patients with non-small-cell lung cancer profiled with targeted next-generation sequencing. J Clin Oncol 2018;36:633-41. doi:10.1200/ JCO.2017.75.3384

10 Hellmann MD, Nathanson T, Rizvi H, et al. Genomic features of response to combination immunotherapy in patients with advanced non-small-cell lung cancer. Cancer Cell 2018;33:843-52. doi:10.1016/j.ccell.2018.03.018

11 Reuben A, Zhang J, Chiou S-H, et al. Comprehensive T cell repertoire characterization of non-small cell lung cancer. Nat Commun 2020;11:603.

12 Biton J, Mansuet-Lupo A, Pécuchet N, et al. TP53, STK11, and EGFR mutations predict tumor immune profile and the response to AntiPD-1 in Lung adenocarcinoma. Clin Cancer Res 2018;24:5710-23. doi:10.1158/1078-0432.CCR-18-0163

13 Gainor JF, Shaw AT, Sequist LV, et al. EGFR mutations and ALK rearrangements are associated with low response rates to PD-1 pathway blockade in non-small cell lung cancer: a retrospective analysis. Clin Cancer Res 2016;22:4585-93. doi:10.1158/1078-0432. CCR-15-3101

14 Spigel DR, Reynolds C, Waterhouse D, et al. Phase 1/2 study of the safety and tolerability of nivolumab plus crizotinib for the first-line treatment of anaplastic lymphoma kinase translocation - positive advanced non-small cell lung cancer (CheckMate 370). J Thorac Oncol 2018;13:682-8. doi:10.1016/j.jtho.2018.02.022

15 Mazieres J, Drilon A, Lusque A, et al. Immune checkpoint inhibitors for patients with advanced lung cancer and oncogenic driver alterations: results from the IMMUNOTARGET registry. Ann Oncol 2019;30:1321-8.

16 Chalmers ZR, Connelly CF, Fabrizio D, et al. Analysis of 100,000 human cancer genomes reveals the landscape of tumor mutational burden. Genome Med 2017;9:34. doi:10.1186/s13073-017-0424-2

17 Gandara DR, Paul SM, Kowanetz M, et al. Blood-Based tumor mutational burden as a predictor of clinical benefit in non-smallcell lung cancer patients treated with atezolizumab. Nat Med 2018:24:1441-8. doi:10.1038/s41591-018-0134-3

18 Dolled-Filhart M, Roach C, Toland G, et al. Development of a companion diagnostic for pembrolizumab in non-small cell lung cancer using immunohistochemistry for programmed death ligand-1. Arch Pathol Lab Med 2016;140:1243-9.

19 Klein JP, Moeschberger ML. Survival analysis: techniques for censored and truncated data. 2nd ed. Berlin: Springer Science \& Business Media, 2005.

20 Zer A, Ding K, Lee SM, et al. Pooled analysis of the prognostic and predictive value of KRAS mutation status and mutation subtype in patients with Non-Small cell lung cancer treated with epidermal growth factor receptor tyrosine kinase inhibitors. Journal of Thoracic Oncology 2016;11:312-23. doi:10.1016/j.jtho.2015.11.010

21 Shepherd FA, Domerg C, Hainaut P, et al. Pooled analysis of the prognostic and predictive effects of KRAS mutation status and KRAS mutation subtype in early-stage resected non-small-cell lung cancer in four trials of adjuvant chemotherapy. $J$ Clin Oncol 2013;31:2173-81.

22 Fung AS, Karimi M, Michiels S, et al. Prognostic and predictive effect of KRAS gene copy number and mutation status in early stage non-small cell lung cancer patients. Transl Lung Cancer Res 2021;10:826-38.

23 Tomasini P, Mascaux C, Jao K, et al. Effect of coexisting KRAS and TP53 mutations in patients treated with chemotherapy for non-smallcell lung cancer. Clin Lung Cancer 2019;20:e338-45. 
24 Rittmeyer A, Barlesi F, Waterkamp D, et al. Atezolizumab versus docetaxel in patients with previously treated non-small-cell lung cancer (oak): a phase 3, open-label, multicentre randomised controlled trial. Lancet 2017;389:255-65.

25 Hong L, Negrao MV, Dibaj SS, et al. Programmed Death-Ligand 1 heterogeneity and its impact on benefit from immune checkpoint inhibitors in NSCLC. J Thorac Oncol 2020;15:1449-59.

26 Jordan EJ, Kim HR, Arcila ME, et al. Prospective comprehensive molecular characterization of lung adenocarcinomas for efficient patient matching to Approved and emerging therapies. Cancer Discov 2017;7:596-609.

27 Le X, Negrao MV, Reuben A, et al. Characterization of the immune landscape of EGFR-mutant NSCLC identifies CD73/adenosine pathway as a potential therapeutic target. $J$ Thorac Oncol 2021:16:583-600.

28 Dudnik E, Peled N, Nechushtan $\mathrm{H}$, et al. Braf mutant lung cancer: programmed death ligand 1 expression, tumor mutational burden, microsatellite instability status, and response to immune CheckPoint inhibitors. J Thorac Oncol 2018;13:1128-37. doi:10.1016/j. jtho.2018.04.024

29 Sabari JK, Leonardi GC, Shu CA, et al. Pd-L1 expression, tumor mutational burden, and response to immunotherapy in patients with Met exon 14 altered lung cancers. Ann Oncol 2018;29:2085-91.

30 Liu C, Peng W, Xu C, et al. Braf inhibition increases tumor infiltration by $T$ cells and enhances the antitumor activity of adoptive immunotherapy in mice. Clin Cancer Res 2013;19:393-403.

31 Ebert PJR, Cheung J, Yang Y, et al. Map kinase inhibition promotes $\mathrm{T}$ cell and anti-tumor activity in combination with PD-L1 checkpoint blockade. Immunity 2016;44:609-21. doi:10.1016/j. immuni.2016.01.024

32 Ascierto PA, Ferrucci PF, Fisher R, et al. Dabrafenib, trametinib and pembrolizumab or placebo in BRAF-mutant melanoma. Nat Med 2019;25:941-6. doi:10.1038/s41591-019-0448-9

33 Gutzmer R, Stroyakovskiy D, Gogas H, et al. Atezolizumab, vemurafenib, and cobimetinib as first-line treatment for unresectable advanced BRAF ${ }^{\mathrm{V} 600}$ mutation-positive melanoma (IMspire150): primary analysis of the randomised, double-blind, placebocontrolled, phase 3 trial. Lancet 2020;395:1835-44. doi:10.1016/ S0140-6736(20)30934-X

34 Skoulidis F, Byers LA, Diao L, et al. Co-Occurring genomic alterations define major subsets of KRAS-mutant lung adenocarcinoma with distinct biology, immune profiles, and therapeutic vulnerabilities. Cancer Discov 2015;5:860-77.

35 Arbour KC, Jordan E, Kim HR, et al. Effects of co-occurring genomic alterations on outcomes in patients with KRAS-mutant non-small cell Lung Cancer. Clin Cancer Res 2018;24:334-40.

36 Skoulidis F, Goldberg ME, Greenawalt DM, et al. STK11/LKB1 mutations and PD-1 inhibitor resistance in KRAS-mutant Lung adenocarcinoma. Cancer Discov 2018;8:822-35.

37 Skoulidis F, Arbour KC, Hellmann MD, et al. Association of STK11/ LKB1 genomic alterations with lack of benefit from the addition of pembrolizumab to platinum doublet chemotherapy in non-squamous non-small cell lung cancer. Journal of Clinical Oncology 2019;37:102. doi:10.1200/JCO.2019.37.15 suppl.102

38 Canon J, Rex K, Saiki AY, et al. The clinical KRAS(G12C) inhibitor AMG 510 drives anti-tumour immunity. Nature 2019;575:217-23.

39 Garassino MC, Cho B-C, Kim J-H, et al. Durvalumab as thirdline or later treatment for advanced non-small-cell lung cancer (Atlantic): an open-label, single-arm, phase 2 study. Lancet Oncol 2018;19:521-36. doi:10.1016/S1470-2045(18)30144-X

40 Lisberg A, Cummings A, Goldman JW, et al. A phase II study of pembrolizumab in EGFR-mutant, PD-L1+, tyrosine kinase inhibitor naïve patients with advanced NSCLC. J Thorac Oncol 2018;13:1138-45.

41 Schoenfeld AJ, Arbour KC, Rizvi H, et al. Severe immune-related adverse events are common with sequential PD-(L)1 blockade and osimertinib. Ann Oncol 2019;30:839-44. doi:10.1093/annonc/ $\mathrm{mdz077}$ 